\title{
IDENTIFICACIÓN Y CUANTIFICACIÓN DEL POTENCIAL DE ENERGÍA UNDIMOTRIZ EN LA COSTA DEL DEPARTAMENTO DEL ATLÁNTICO, COLOMBIA
}

\author{
Identification and quantification of potential wave energy in the coast of Atlántico state in \\ Colombia
}

\author{
Jaime Pérez-Zapata ${ }^{1}$ \\ ${ }^{1}$ Ing., Institución Universitaria ITSA. (Soledad-Atlántico, Colombia). jperezz@itsa.edu.co
}

(Recibido mayo 30 de 2017 y Aprobado junio 10 de 2017)

\begin{abstract}
Resumen
Este documento describe los avances realizados con relación a la identificación y cuantificación del potencial de energía undimotriz en las costas del departamento del Atlántico. La metodología utilizada servirá como punto de partida para establecer el potencial que tiene Colombia en este tipo de fuente no convencional de energía renovable. Se estimó que el potencial energético cercano a la costa (near shore) teórico que poseen las olas de las costas del departamento del Atlántico es de $1.107 \mathrm{MW}$ aproximadamente, lo cual fue posible gracias a los datos que han sido recopilados y procesados desde el mes de enero 2016, es decir, con un historial de datos mayor a un año, dándole mayor confiabilidad a los cálculos realizados. Fueron aplicadas ecuaciones consultadas, las cuales tienen en cuenta variables como altura significativa de la ola, periodo, densidad del agua de mar, temperatura y velocidad del viento en la zona. Así mismo se muestra el avance del diseño de un sistema near shore para la verificación del potencial energético, que consiste en un dispositivo que transforma la energía cinética de las olas del mar en energía mecánica, para bombear agua a una determinada altura convirtiéndola en energía potencial gravitacional y que posteriormente mueva un generador de corriente alterna.
\end{abstract}

Palabras clave: energía undimotriz; altura significativa; periodo; potencial de energía undimotriz; boya oceanográfica; frente de ola.

\begin{abstract}
This paper describes the progress regarding the identification and quantification of potential wave energy in Atlántico state coast. The used methodology will serve as a starting point to establish the energetic potential in Colombia in this energy. The theoretical energetic potential estimated at the near shore in the Atlantic coast waves is approximately 1107 MW and this was possible with data that has been collected and processed since January 2016. We applied equations that take into account variables such as: significant wave height, period, sea water density, temperature and wind speed in the area. Also it is shown the progress of the near shore system design for the verification of the wave energetic potential consisting of a device which transforms kinetic energy of the sea waves into gravitational potential energy that is going to be used to move an alternating current generator.
\end{abstract}

Keywords: wave energy; significant wave height, wave period; wave power potential; oceanographic buoy; wave front; point absorbers, on shore; near shore; off shore.

\section{INTRODUCCIÓN}

Este documento describe los avances realizados con relación a la identificación y cuantificación del potencial de energía undimotriz (aquella que se obtiene mediante las olas del mar) [1] en las costas del departamento del Atlántico. La metodología utilizada servirá como punto de partida para establecer el potencial que tiene Colombia en este tipo de fuente no convencional de energía renovable (FNCER) y con esto atraer el interés por parte de la comunidad investigadora nacional e internacional en la implementación de tecnologías que aprovechen este tipo de energía primaria para convertirla en energía eléctrica. Con esto se logra, de 
igual manera, realizar transferencia de conocimientos y de tecnología.

En Colombia, la energía undimotriz es poco conocida y desarrollada, además ha sido poco investigada, desconociendo el gran potencial energético que poseen las olas, especialmente en un país como este, que posee una gran extensión de costas.

Para cumplir con el objetivo se requiere conocer el potencial energético que poseen las olas y esto fue posible con la aplicación de ecuaciones consultadas las cuales tienen en cuenta variables como: altura de la ola, periodo de las olas, densidad del agua de mar, temperatura y velocidad del viento en la zona. Estos datos han sido recopilados en conjunto con la Dirección General Marítima (DIMAR) y el Centro de Investigaciones Oceanográficas $(\mathrm{ClOH})$ adscritas al Ministerio de Defensa Nacional (Mindefensa)[2]. Los datos han sido recopilados y procesados desde el mes de enero de 2014, es decir, con un historial de datos mayor de tres años. Si bien es cierto que se requiere de un mayor historial de datos, los obtenidos hasta el momento son los que se encuentran disponibles en las bases de datos del $\mathrm{ClOH}$. Consideramos que los datos procesados dan confiabilidad a los cálculos realizados para la determinación del potencial de energía undimotriz de la zona objetivo, dado que el comportamiento de las olas en el área de estudio muestra poca variabilidad de un año a otro. No se efectúan comparaciones con otras zonas costeras colombianas, por los pocos estudios realizados con relación a las características de las olas y mucho menos sobre su potencial energético; además, aquí se pretende estudiar solamente el comportamiento de las olas en las costas del departamento del Atlántico, Colombia.

\section{CARACTERIZACIÓN DE LAS OLAS EN LAS COSTAS DEL DEPARTAMENTO DEL ATLÁNTICO}

Tomando como base la teoría de estudios anteriormente realizados, las olas poseen las características que se muestran en la Figura 1:

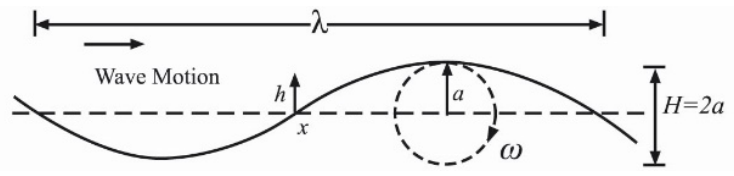

Figura. 1. Parámetros de una onda [3].
La diferencia de altura entre la cresta y el valle es la altura de la ola $\mathrm{H}$, y la amplitud es la mitad de la altura; la longitud de la onda $\lambda$ es la distancia entre 2 crestas consecutivas, y $\omega$ es la frecuencia angular [3], la cual se muestra en la ecuación (1):

$\omega=\frac{2 \pi}{\tau}$

Donde $\pi$ es una constante matemática aproximadamente igual a 3,1416 y $\mathrm{T}$ es el periodo de la onda, el periodo es el tiempo que una onda tarda en recorrer toda su longitud [3].

Otro parámetro de las ondas es su velocidad, también denominada velocidad de fase, que es la velocidad con la que se mueve la onda [1]:

$c=\frac{\lambda}{\tau}$

La velocidad de fase cambia en relación con la profundidad del mar sobre la cual pasan las olas[3]. Para aguas profundas (donde la profundidad es mayor a la mitad de la longitud de onda), la velocidad de fase es:

$c=\frac{g}{2 \pi} T$

Para aguas poco profundas, donde la profundidad del mar es menor a ${ }_{20}^{1 /}$ la longitud de la onda (o la longitud de la onda multiplicada por 0,05 ) la velocidad de fase es igual a [3]:

$c=\sqrt{g H}$

Donde $g$ es la fuerza de gravedad $\left(9,80665 \mathrm{~m} / \mathrm{s}^{2}\right)$ y H es la altura de la ola.

Cuando la profundidad del mar es mayor a la mitad de la longitud de onda y menor que 0,05 por la longitud de la onda las aguas se consideran intermedias. La fórmula de la velocidad de fase de una ola en estas aguas es:

$c=\sqrt{\frac{g}{k} \tan H(k H)}$

Donde $\mathrm{H}$ es la altura de la ola, $g$ es la gravedad y $k$ es el número de onda. 
Teniendo en cuenta lo anterior, el área de estudio se caracteriza como aguas de poca profundidad.

\section{CUANTIFICACIÓN DEL POTENCIAL DE ENERGÍA UNDIMOTRIZ EN EL DEPARTAMENTO DEL ATLÁNTICO}

Los potenciales relativos de los recursos de la energía disponible en las olas de los océanos en el mundo se encuentran alrededor de $2.700 \mathrm{GW}$ [4], y el total de energía disponible para usarse es de casi $500 \mathrm{GW}$. Se estima que la energía undimotriz total que incide en las costas del mundo sobre la superficie del océano es de más o menos 2,11 TW [5]. De acuerdo con estudios, en el océano Atlántico, en cercanías a las costas colombianas en el mar Caribe, el promedio anual de niveles de energía undimotriz oscila de 10 a $20 \mathrm{~kW} / \mathrm{m}$ de frente de ola [1], como se aprecia en la Figura 2.

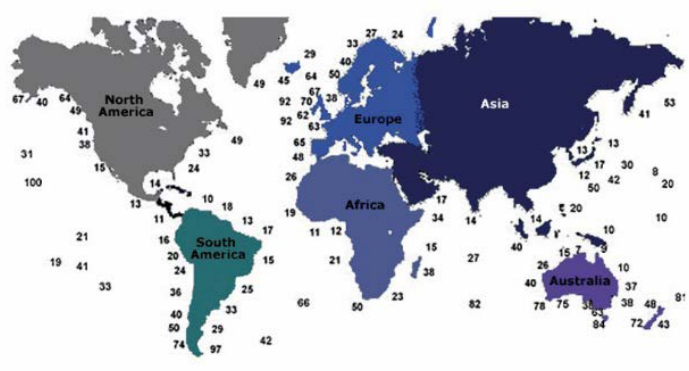

Figura. 2. Potenciales de energía undimotriz en el mundo [6].

Sin embargo, para la elaboración de este documento se hicieron cálculos basados en datos medidos localmente de las variables que intervienen en la cuantificación del potencial energético. Implementamos una metodología que arrojó resultados que son mostrados posteriormente

\subsection{Ecuaciones utilizadas}

Teniendo en cuenta todas las consultas bibliográficas realizadas con relación al tema, se han seleccionado las ecuaciones que se muestran a continuación, con base en las aproximaciones al comportamiento de las olas del mar en la zona delimitada de las costas del departamento del Atlántico, teniendo en cuenta variables como altura significativa y periodo de las mismas. Estas formulaciones pueden ser de igual manera utilizadas para cuantificar el potencial, al menos, en las costas del mar Caribe colombiano.

En un oleaje idealizado, en el cual la altura y el periodo de las olas son constantes, la energía de las olas puede calcularse utilizando la siguiente formula [7]:

$P=\frac{\rho g^{2} H^{2} T}{32 \pi}$

Donde: P: potencia (W), $\rho$ : densidad del agua (en este caso agua salada de mar): $1.025 \mathrm{~kg} / \mathrm{m3}$, g: gravedad $(9.8 \mathrm{~m} / \mathrm{s} 2) \mathrm{T}$ : periodo de la ola $(\mathrm{s}), \mathrm{H}$ : altura promedio de la ola $(\mathrm{m})$. Sin embargo, el típico estado del mar está generalmente compuesto por olas que poseen diferentes alturas, periodos y direcciones. Para un mar irregular, la energía total promedio que se encuentra en las olas se calcula [3]:

$P=k H_{s}^{2} T_{e}$

Donde $k$ es una constante que viene dada por la siguiente ecuación [4]:

$k=\frac{\rho g^{2}}{64 \pi}$

Donde $\rho$ es la densidad del agua de $\operatorname{mar}\left(1025 \mathrm{~kg} / \mathrm{m}^{3}\right)$, es la gravedad $\left(9.80665 \mathrm{~m} / \mathrm{s}^{2}\right)$ у л es una constante matemática cuyo valor aproximado es igual a 3.1416. Como $k$ es una constante, y se conocen sus variables, basta con hallar su valor y reemplazarlo para que la ecuación de la energía undimotriz quede de la siguiente manera:

$P=490,27 H_{s}^{2} T_{e} \approx 0,49 H_{s}^{2} T_{e}$

Donde $P$ es el flujo de energía de la ola expresado en $\mathrm{kW} / \mathrm{m}, H_{s}$ es la altura significativa de la ola y $T e$ es el período en segundos. 
La altura significativa $H_{s^{\prime}}$ se define como la altura promedio del tercio de olas más altas dentro de un grupo de olas considerado. Este valor se aproxima a la altura que un observador en una embarcación reportara de una inspección visual del estado del mar, ya que tal observador tiende a omitir las olas más pequeñas y menos evidentes. El periodo de energía Te es el periodo de ola promedio con respecto al espectro de distribución de transporte de energía de las olas [5].

Los mares reales contienen olas que poseen alturas, periodos y direcciones aleatorios. Dentro de un corto periodo de tiempo, las características de los mares reales permanecen constantes, componiéndose así de un estado del mar [5]. Con el fin de describir estos estados del mar y determinar sus características relevantes para los dispositivos de energía undimotriz, se utilizan parámetros estáticos antes mencionados, los cuales se pueden obtener utilizando dispositivos de medida de las características de las olas, como las boyas oceanográficas o también conocidas como boyas de oleaje [6].

La ecuación (9) se utilizó para realizar las primeras estimaciones del posible potencial de energía undimotriz near shore que tienen las costas del departamento del Atlántico.

\subsection{Datos obtenidos}

Los datos necesarios para aplicarlos en la ecuación seleccionada se obtuvieron de la base de datos disponible en el $\mathrm{ClOH}[2]$, la cual es una dependencia de DIMAR y estas forman parte del Mindefensa.

Para poder conocer las características del oleaje anteriormente descrito, se usan las denominadas boyas oceanográficas, las cuales son utilizadas para registrar las diferentes variables océano-atmosféricas que pueda haber en los mares, como la altura y dirección de las olas, así como su periodo, la dirección y fuerza del viento, la temperatura tanto del agua como del aire, la presión atmosférica, entre otras.

Estos dispositivos constan de un ordenador que se encarga de recoger la información que obtienen de sus medidores en tiempo real y la envían a través de un sistema de comunicación inalámbrico hacia una estación receptora en tierra para su posterior análisis [6].

Para abastecer de energía los componentes de la boya se utilizan paneles fotovoltaicos, los cuales están protegidos del mar y el viento por medio de una cúpula de vidrio. Por otro lado, la boya se encuentra anclada al fondo marino, como se puede ver en la Figura 3, y en caso de soltarse, posee un sistema de rastreo que permite conocer su ubicación en tiempo real [6].

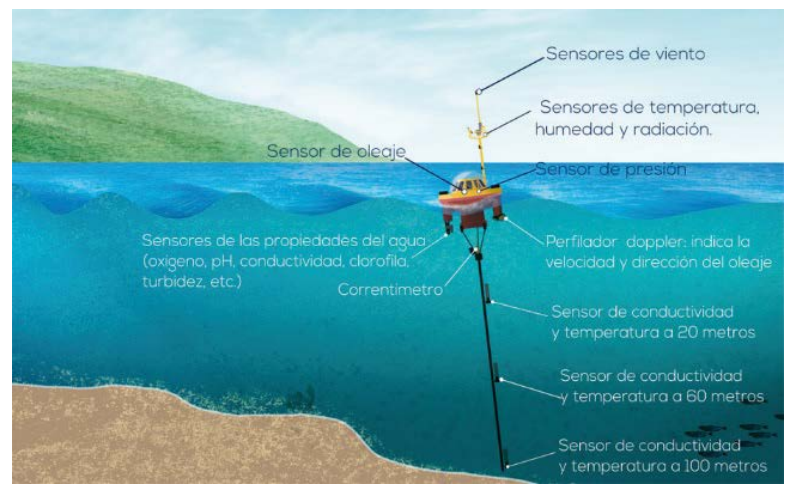

Figura. 3. Esquema de una boya oceanográfica [8].

$\mathrm{El} \mathrm{ClOH}$ cuenta con cuatro boyas oceanográficas ubicadas en el mar Caribe de la siguiente manera: una en cercanías a las islas de San Andrés y Providencia, otra cerca de Puerto Bolívar en la Guajira, otra en cercanías de Barranquilla y otra cerca de Coveñas, como se muestra en la Figura 4.

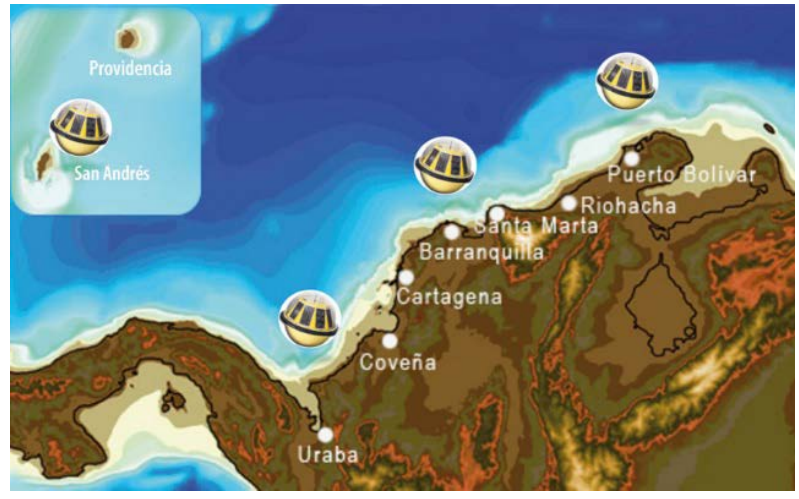

Figura. 4. Ubicación boyas en la costa atlántica[2].

Para la zona objetivo se recopilaron datos suministrados por el $\mathrm{ClOH}$, registrados por las boyas de oleaje ubicadas en diferentes zonas de la costa del departamento del Atlántico. Como ejemplo, en las Tablas 1 y 2 se muestra información sobre las características del olaje en diferentes zonas durante algunos días del mes de septiembre del 2016. 
Tabla 1. Oleaje en el Muelle [2].

\begin{tabular}{lll}
\hline Día & Oleaje $(\mathrm{m})$ & Periodo $(\mathrm{s})$ \\
\hline 13 & 0.4 & 6.25 \\
14 & 0.51 & 5.87 \\
15 & 0.6 & 6.87 \\
16 & 0.52 & 6.75 \\
17 & 0.71 & 6.5 \\
18 & 0.7 & 7 \\
19 & 0.71 & 7 \\
20 & 0.55 & 7 \\
\hline
\end{tabular}

Tabla 1. Oleaje en "El Bolsillo" Pradomar [2].

\begin{tabular}{lcl}
\hline Día & Oleaje $(\mathrm{m})$ & Periodo $(\mathrm{s})$ \\
\hline 13 & 0.44 & 6.25 \\
14 & 0.55 & 5.87 \\
15 & 0.66 & 6.87 \\
16 & 0.54 & 6.75 \\
17 & 0.77 & 6.5 \\
18 & 0.82 & 7 \\
19 & 0.74 & 7 \\
20 & 0.6 & 7 \\
\hline
\end{tabular}

Se cuenta con datos de otras zonas pertenecientes a las costas del departamento del Atlántico como son: oleaje en "Punta Roca" (Salgar), oleaje en "Puerto Velero" y Ooeaje en "Charco Grande". Esta información puede ser consultada en la base de datos del $\mathrm{ClOH}$.

Para establecer un oleaje promedio en la zona objetivo y con el fin de obtener información más generalizada del oleaje, además de darle mayor confiabilidad a los cálculos realizados, es necesario hacer un seguimiento al oleaje durante un periodo más amplio que el mostrado anteriormente. Es por esto que para los cálculos de este estudio se tuvo en cuenta la altura que podían alcanzar las olas en un día dado, entre las fechas del 1 de enero de 2016 y el 31 de enero de 2017 (es decir, durante 13 meses) periodo que se tomó como muestra. Con dicha información se obtuvo la altura significativa del oleaje presente en cada mes, de esta forma se puede conseguir mayor precisión en el conocimiento del comportamiento del oleaje. La información utilizada para llevar a cabo lo anteriormente descrito se puede encontrar (día por día) en el sitio web del $\mathrm{ClOH}$, así como también otros datos acerca del mar y el clima en la costa Caribe colombiana. Como ejemplo, en las
Figuras 5 y 6 se muestra la altura promedio calculada del oleaje registrado en los meses de enero y diciembre 2016:

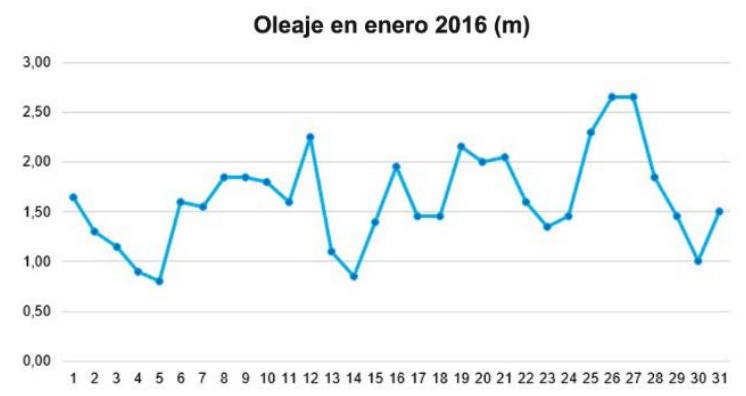

Figura 5. Oleaje promedio calculado diario mes de enero 2016.

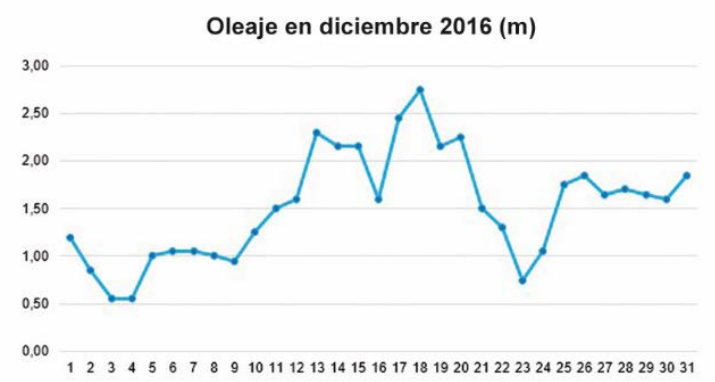

Figura 6. Oleaje promedio calculado diario mes de diciembre 2016.

\subsection{Cálculos del potencial de energía undimotriz}

Después de haber conseguido la altura promedio calculada con base en los datos obtenidos, procedimos a estimar la altura significativa $\mathrm{Hs}$ aplicando el concepto anteriormente descrito. Se tomó el tercio de los datos correspondiente a un mes de los resultados de altura de olas promedio más alto. En la Figura 7 se muestra la altura significativa calculada para los 13 meses de registro de datos.

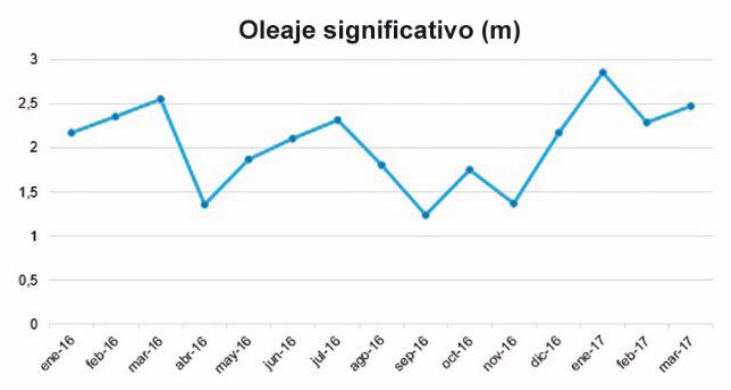

Figura. 7. Altura significativa del oleaje en el Atlántico. 
Como se puede observar, el comportamiento del oleaje varía de acuerdo con la época del año, por ende, el potencial energético también.

Usando la ecuación (9) del potencial de energía undimotriz anteriormente descrito, se puede hacer una aproximación de lo que sería el potencial de energía undimotriz teórico presente en las costas del Atlántico. La ecuación (9) tiene en cuenta el periodo de la ola, por lo que con base en el periodo registrado en distintos puntos de la zona y para fines prácticos se utiliza un periodo de 6 segundos. Como ejemplo, para el mes de enero del 2016 , la potencia ofrecida $(\mathrm{kW} / \mathrm{m})$ por el oleaje se calculó de la siguiente manera:

$P=0,49 H_{s}^{2} T$

$P=0,49 \times(2.17 m)^{2} \times 6 s$

$P=13,84 \mathrm{~kW}$

En la Tabla 3 se muestran los cálculos de potencia de frente de ola realizados teniendo en cuenta la altura significativa $\mathrm{Hs}$ de cada mes.

Tabla 3. Potencia de frente de ola estimada.

\begin{tabular}{lcc}
\hline \multicolumn{1}{c}{ Mes } & $\begin{array}{c}\text { Altura } \\
\text { significativa }(\mathrm{m})\end{array}$ & Potencia (kW/m) \\
\hline Enero 2016 & 2.17 & 13.84 \\
Febrero 2016 & 2.35 & 16.24 \\
Marzo 2016 & 2.55 & 19.12 \\
Abril 2016 & 1.36 & 5.44 \\
Mayo 2016 & 1.87 & 10.28 \\
Junio 2016 & 2.11 & 13.09 \\
Julio 2016 & 2.31 & 15.69 \\
Agosto 2016 & 1.8 & 9.53 \\
Septiembre 2016 & 1.24 & 4.52 \\
Octubre 2016 & 1.75 & 9.00 \\
Noviembre 2016 & 1.37 & 5.52 \\
Diciembre 2016 & 2.17 & 13.84 \\
Enero 2017 & 2.85 & 23.88 \\
\hline
\end{tabular}

Así, analizando los datos obtenidos en la tabla anterior, los mayores potenciales de energía de las olas están presentes entre los meses de enero y marzo, los meses de mayo hasta julio y en el mes de diciembre. Cabe destacar que en todos los meses se cuenta con un considerable potencial energético.
Con los anteriores datos se calcula la potencia total promedio de frente de ola de los meses estimados, que da como resultado $12,3 \mathrm{~kW} / \mathrm{m}$. Si tenemos en cuenta la extensión de la costa del Atlántico, la cual es de $90 \mathrm{~km}$, daría como resultado un potencial teórico aproximado de 1'107.000 kW o mejor $1.107 \mathrm{MW}$ para aplicación de tecnología near shore. Para lo anterior, se deben realizar algunos ajustes bajo la premisa de que no sería posible la instalación de tecnología aplicada al aprovechamiento de la energía undimotriz en toda la extensión de la costa, ya que esto dependería de las políticas ambientales locales y de la selectividad de las zonas con mayor potencial. También deben tenerse en cuenta las eficiencias de los equipos de conversión de energía undimotriz para ajustar el potencial calculado que dependerá de la tecnología escogida. Debemos considerar que este potencial puede aumentar si se aplica tecnología on shore y off shore simultáneamente, las cuales no han sido examinadas aún en este estudio.

\section{AVANCES EN LA VERIFICACIÓN DEL POTENCIAL ENERGÉTICO}

Con el objetivo de demostrar cuantitativamente el potencial energético contenido en las olas del mar presentadas en el departamento del Atlántico, se está trabajando en el diseño de un sistema de aprovechamiento near shore, el cual forma parte de las tecnologías denominadas point absorbers. Los dispositivos point absorbers son estructuras flotantes con componentes que se mueven debido a la acción de la ola, estos dispositivos han sido de los más investigados desde el comienzo del desarrollo de la energía undimotriz.

Los point absorbers utilizan boyas que se mueven junto con las olas, el movimiento relativo hacia arriba y hacia abajo de las olas que pasan para que conversores de energía electromecánica o hidráulica generen energía. La forma de la boya no tiene un impacto significativo a la hora de absorber la energía de las olas, debido a que una boya con un tamaño razonable posee un periodo de oscilación mayor que la frecuencia de las olas. Entre más grande sea la boya, más energía puede absorber del mar, sin embargo, si la boya es demasiado grande, la ola no será capaz de pasar sobre ella y no hará funcionar al dispositivo point absorber. De este modo, para poder hacer buen uso de este tipo de dispositivo es necesario elaborar un diseño que absorba la mayor cantidad de energía que le sea posible, teniendo en cuenta el 
tamaño de la boya, así como también otros factores [9]. Si el periodo de oscilación del dispositivo point absorber coincide con el de las olas que en el inciden, podrá absorber y enviar más energía de las olas hacia el sistema de extracción de energía.

El sistema de aprovechamiento que se está diseñando consiste en un dispositivo de este tipo, el cual transforma la energía cinética de las olas del mar en energía mecánica, a fin de bombear agua a una determinada altura convirtiéndola en energía potencial gravitacional, para que posteriormente se deje caer y mueva un generador. Se puede apreciar el esquema del sistema en la Figura 8.

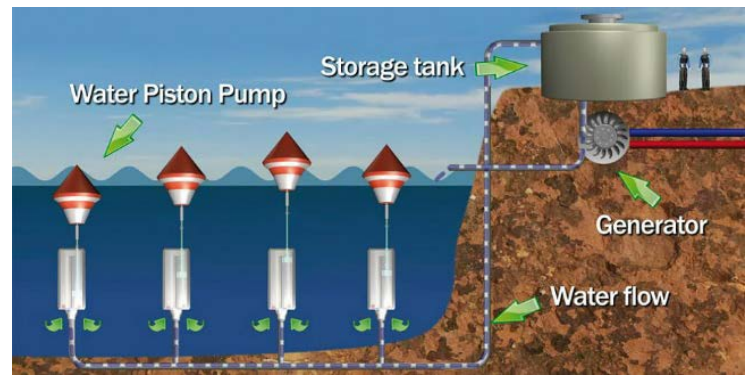

Figura 8. Esquema del sistema en etapa de diseño [10].

Actualmente nos encontramos en la etapa de diseño de la bomba hidráulica que bombeará el agua de mar a una determinada altura, esta será diseñada a escala. Lo anterior permitirá medir el potencial energético real en las costas del Atlántico, así como también sentar las bases para el diseño de un sistema de captación a mayor escala. En la Figura 9 se muestra el diseño de la bomba.

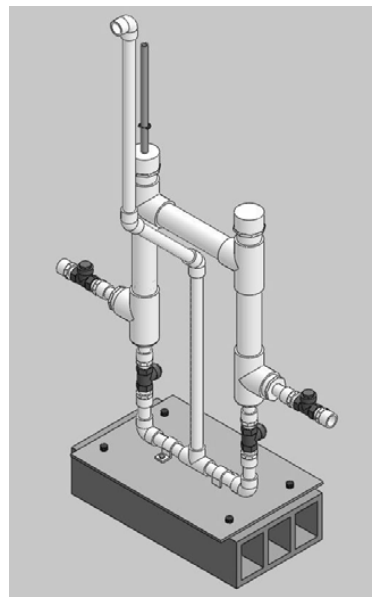

Figura 9.Diseño bomba hidráulica accionada por boya.
La bomba está constituida principalmente por 2 cilindros de 7,5 pulgadas (19,05 centímetros) de largo y 1,5 pulgadas (3,81 centímetros) de diámetro, los cuales están unidos en la parte de arriba por una tubería. En uno de los cilindros se encuentran un vástago de 19,68 pulgadas (50 centímetros) de largo y un émbolo. EI vástago y el émbolo están unidos a una boya calculada de $6 \mathrm{~kg}$ con una geometría definida, esta es la que se encarga de bombear el agua hacia las tuberías. A los costados de ambos cilindros hay un agujero en el cual se aloja una válvula unidireccional, que es la que permite el paso del agua hacia ambos cilindros. La intención de la misma es que con el movimiento de las olas (ascendente y descendente) la boya pueda ejercer la presión continua suficiente para bombear el agua de mar hasta un tanque de almacenamiento, el cual se encontrará ubicado a una determinada altura. Cuando el tanque esté lleno hasta cierto nivel, se dejará caer el agua a través de un tramo de tuberías para que mueva un generador de corriente alterna y, de esta forma, generar energía eléctrica.

\subsection{Cálculo de la potencia de la bomba hidráulica}

La potencia que la bomba obtendrá de las olas del mar para bombear el agua hasta el tanque elevado se puede estimar con la utilización de la siguiente ecuación[11]:

$P=\gamma \times Q \times H_{t}$

Donde $\mathrm{P}$ es la potencia de la bomba, en vatios (W), $\gamma$ es el peso específico del líquido que se va a bombear. El peso específico se halla multiplicando la densidad del líquido, que en este caso es agua de mar $(1025 \mathrm{Kg} /$ $\left.\mathrm{m}^{3}\right)$ por la gravedad $\left(9,80665 \mathrm{~m} / \mathrm{s}^{2}\right)$. Esta operación da como resultado un peso específico del agua de mar de $10051,81 \mathrm{~N} / \mathrm{m}^{3}$, Q es el caudal, el cual posee un valor medido de $0,000145092 \mathrm{~m}^{3} / \mathrm{s}$, y $\mathrm{H}_{\mathrm{t}}$ es la altura total a la que se va a elevar el agua bombeada.

La altura total de elevación se calcula con la siguiente ecuación [8]:

$H_{t}=H_{a}+H_{f}$

Donde $\mathrm{H}_{\mathrm{e}}$ es la altura de elevación del agua, que para este diseño será de 4 metros $(\mathrm{m})$, y $\mathrm{H}_{\mathrm{f}}$ son las pérdidas de carga totales que hay en las tuberías, en metros ( $m$ ). 
Para hallar las pérdidas totales se tienen en cuenta dos tipos de pérdidas, las generales, que son las que se encuentran en la tubería de conducción, y las pérdidas específicas, que se encuentran en los accesorios que forman parte de la tubería de conducción.

Hallamos primero las generales, para ello se utiliza la ecuación de Darcy-Weisbach [8]:

$H_{g}=f \times\left(\frac{L_{c}}{D}\right) \times\left(\frac{v^{2}}{2 g}\right)$

Donde $H_{g}$ son las pérdidas de carga en la tubería de conducción, debido a la fricción del agua dentro de esta, expresadas en metros, $f$ es el coeficiente de fricción de Darcy-weisbach, un valor adimensional que se calcula teniendo en cuenta el número de Reynolds y la rugosidad relativa. Para este diseño, el coeficiente de fricción posee un valor de $0.022, \mathrm{~L}_{\mathrm{c}}$ es la longitud de la tubería de conducción en metros. En este diseño se utilizará un tramo de tubería de conducción de 35 metros de largo, $D$ es el diámetro de la tubería de conducción también en metros, el cual será de 0.0127 metros ( 1.27 centímetros), $v$ es la velocidad del agua en la tubería de conducción en metros por segundo $(\mathrm{m} / \mathrm{s})$ y g es la gravedad.

Hallamos la velocidad, que es el valor que hace falta, de la siguiente forma:

$v=\frac{Q}{A}$

Donde $Q$ es el caudal en la tubería de conducción y $A$ es el área de esa misma tubería, el cual es igual a:

$A=\frac{\pi}{4} \times D^{2}$

$A=\frac{\pi}{4} \times(0,0127 m)^{2}$

$A=0,000126 \mathrm{~m}^{2}$

Ahora, reemplazamos en la ecuación (14) de la velocidad del agua en las tuberías:

$v=\frac{0,000145092 \mathrm{~m}^{3} / \mathrm{s}}{0,000126 \mathrm{~m}^{2}}$ $v=1,15 \mathrm{~m} / \mathrm{s}$

Como ya se tiene la velocidad, se pueden reemplazar los valores de la ecuación (13) de Darcy-weisbach:

$H_{f}=0,022 \times\left(\frac{35 \mathrm{~m}}{0,0127 \mathrm{~m}}\right) \times\left(\frac{\left(1,15 \frac{\mathrm{m}}{\mathrm{s}}\right)^{2}}{2 \times 9,80665 \mathrm{~m} / \mathrm{s}^{2}}\right)$

$H_{g}=0,022 \times 2755,9 \mathrm{~m}^{2} \times 0,0674 \mathrm{~m}$

$H_{g}=4,08 m$

Las pérdidas en la tubería de conducción debido a la fricción son de 4.08 metros; a estas pérdidas generales se le suman las pérdidas específicas en los accesorios del tramo de tubería de conducción para tener un valor más exacto de las pérdidas de carga. Las pérdidas de carga en accesorios se calculan con la siguiente ecuación [8]:

$H_{e}=K \times \frac{v^{2}}{2 g}$

Donde $\mathrm{H}_{\varepsilon}$ son las pérdidas específicas en los accesorios de la tubería de conducción, $\mathrm{K}$ es un coeficiente igual a la suma de las longitudes equivalentes a tubería recta de los accesorios del tramo de tubería de conducción. Dicho tramo tendrá un (1) accesorio $T$ de 90 grados y 2 codos de 45 grados, los coeficientes de $\mathrm{K}$ para la T y los codos son 1.38 y 0.86 respectivamente, sumados estos dos valores dan como resultado un coeficiente $\mathrm{K}$ de 2.24. Enseguida se reemplazan los valores en la ecuación (16) de pérdidas:

$H_{a}=2,24 \times \frac{(1,15 \mathrm{~m} / \mathrm{s})^{2}}{2 \times 9,80665 \mathrm{~m} / \mathrm{s}^{2}}$

$H_{a}=2,24 \times 0,0674 \mathrm{~m}$

$H_{a}=0,15 \mathrm{~m}$

Ya con ambas pérdidas, generales y específicas de los accesorios, se calculan las pérdidas totales en la tubería de conducción, para obtener las pérdidas totales y, con estas últimas, hallar la altura total de elevación, reemplazando en la ecuación (12):

$H_{\mathrm{t}}=4 m+(4,08 m+0,15 m)$

$H_{\mathrm{t}}=4 m+4,23 m$ 
$H_{\mathrm{t}}=8,23 \mathrm{~m}$

Conociendo todas las variables necesarias para calcular la potencia de la bomba hidráulica, solo queda reemplazarlas en la ecuación (11) y resolverla:

$P=10051,81 \mathrm{~N} / \mathrm{m}^{3} \times 0,000145092 \mathrm{~m}^{3} / \mathrm{s} \times 8,23 \mathrm{~m}$

$P=12 W$

\subsection{Cálculo del peso de la boya}

Para calcular este valor se utiliza la siguiente ecuación:

Peso $_{\text {boya }}=\left(\left(A_{i} \times D_{c}\right) \times \rho\right)+\left(\left(A_{c} \times L_{c}\right) \times \rho\right)$

Donde:

$\mathrm{A}_{\mathrm{i}}$ = área de la tubería de impulsión, donde se bombea el agua $\left(0,00114 \mathrm{~m}^{2}\right)$

$D_{c}=$ distancia de la camisa de la bomba, desde el émbolo (en su posición más alta) hasta el fondo de camisa, esta distancia es de $22,5 \mathrm{~cm}(0,225 \mathrm{~m})$

$\mathrm{A}_{\mathrm{c}}=$ es el área del tramo de tubería de conducción $\left(0,000126 \mathrm{~m}^{2}\right)$

$\mathrm{L}_{\mathrm{c}}=$ es la longitud del tramo de tubería de conducción (35 m)

$\rho=$ es la densidad del agua de mar

Al multiplicar $A_{i} \times D_{c}$ y $A_{c} \times L_{c}$ obtendremos los volúmenes de la camisa de la bomba y el del tramo de tubería de conducción respectivamente. Sabiendo esto, la ecuación puede simplificarse y quedará como:

$$
\text { Peso boya }_{\text {ba }}=\left(\left(V_{i}\right) \times \rho\right)+\left(\left(V_{c}\right) \times \rho\right)
$$

En la que $V_{i}$ es el volumen de la camisa $\left(0,000256 \mathrm{~m}^{3}\right)$, y $V_{c}$ es el volumen del tramo de tubería de conducción $\left(0,00441 \mathrm{~m}^{3}\right)$ :

Peso $_{\text {boya }}=\left(0,000256 \mathrm{~m}^{3} \times 1025 \mathrm{~kg} / \mathrm{m}^{3}\right)+$

$$
\begin{aligned}
& +\left(0,00441 \mathrm{~m}^{3} \times 1025 \mathrm{~kg} / \mathrm{m}^{3}\right) \\
& \text { Peso }_{\text {boya }}=(0,26 \mathrm{Kg})+(4,52 \mathrm{~kg}) \\
& \text { Peso boya }_{\text {bo }}=4,78 \mathrm{~kg}
\end{aligned}
$$

De acuerdo con el resultado, la boya debe tener un peso mínimo de 4,78 kg, aunque preferiblemente el peso de la boya deberá ser mayor al resultado de la fórmula, para garantizar un funcionamiento óptimo de la bomba hidráulica. Por esta razón y por efectos prácticos se ha decidido que la boya para el dispositivo será de $6,6 \mathrm{~kg}$ de peso, por encontrar en el mercado pesas calibradas de $2,2 \mathrm{~kg}$.

\section{CONCLUSIONES}

Es evidente que existe un potencial de energía undimotriz en las costas del Caribe colombiano y, para que pueda ser aprovechado, es importante dar el primer paso en su identificación y cuantificación. Apoyándonos en los resultados obtenidos, en las costas del departamento del Atlántico, el potencial de energía undimotriz teórico estimado resulta interesante para la aplicación de tecnologías que aprovechen este tipo de energía. Si bien los datos nos orientan a la aplicación de tecnologías near shore, estos nos pueden servir como referencia para las aplicaciones on shore $y$ off shore, aumentando el potencial estimado. De acuerdo con los cálculos realizados para la bomba hidráulica a escala que se propone, se dan unos resultados que nos indican la factibilidad de obtener energía eléctrica en las olas de las costas del Atlántico, teniendo en cuenta las características del oleaje presentado.

\section{REFERENCIAS}

[1] J. Brooke, Wave Energy Conversion. Annapolis: Elsevier Science Ltd., 2003.

[2] Cloh. org (2017). ClOH Oceanografía Operacional 2017. [En línea]. Available: https://www.cioh.org. co/meteorologia/index.php.

[3] J. Muñoz, "Ondas regulares y su aplicación a la ingeniería de costas. Cádiz, ES: Publicaciones de la Universidad de Cádiz, 2011, p. 2.

[4] M. McCormick and C. Ertekin, "Renewable sea power", Renew. Energy, vol 131, pp. 36-45, 2009.

[5] K. Gunn and C. Stock-Williams, "Quantifying the global wave power resource", Renew. Energy, 
vol. 44, pp. 296-304, 2012. doi: https://doi.or$\mathrm{g} / 10.1016 / \mathrm{j}$. renene.2012.01.101

[6] Madrimasd.org (s.f.). Ciencias marinas y otros asuntos. [En línea]. Disponible: http://www.madrimasd. org/blogs/ciencia marina/2007/08/20/72161

[7] L. Duckers, "Wave energy ; crests and troughs". Renewable Energy. vol 5, pp 1444-1452, agosto, 1994.

[8] Medclic (s.f.). Infraestructuras fijas. [En línea]. Disponible: http://www.medclic.es/es/instrumentos/ infraestructuras-fijas/

[9] J. Engstrom, "Hydrodynamic Modelling for a point Absorbing Wave Energy Converter," Digit. Compr. Summ. Uppsala Diss. from Fac. Sci. Technol., vol. 878, 2011.

[10] Earthprotect (s.f.). Blue Energy - Ocean Power (Piston Pump \&amp; Racks). [En línea]. Disponible: http://www.earthprotect.com/index.php/me- dia-gallery/mediaitem/3067-blue-energy-oceanpower-piston-pump-racks.

[11] Aula Virtual (s.f.). Proyecto fin de carrera Ingenieria Química. [En línea]. Disponible: http://www.ugr. es/ aulavirtualpfciq/Bbombasytuberias.html.

[12] A. Khaligh y O.C. Onar, "Chapter 4. Ocean Wave Energy Harvesting", Energy Harvesting, A. Emadi, Ed. CRC Press2010: Boca Raton FL, 2010, pp. 223303.

[13] A. F. d. O. Falcão, "Wave Energy Utilization: A Review of the Technologies", Renewable and Sustainable Energy Reviews, vol no 14, pp.899-918,abril,2010. doi: https://doi.org/10.1016/j.rser.2009.11.003

[14] Boreau of Ocean Energy Management BOEM. (s.f.). Ocean wave energy. [En Línea]. Disponible: https://www.boem.gov/Ocean-Wave-Energy/

[15] J. Twidell y T. Weir, Renowable Energy Resources, third edition Routledge: N.Y., pp. 408-434, 2015. 